\title{
EFEITOS DO MANTO DE INTEMPERISMO SOBRE ANOMALIAS ELETROMAGNÉTICAS (HLEM) GERADAS POR CORPOS TABULARES INCLINADOS
}

\author{
LỨCIA MARIA DA COSTA E SILVA * e OM PRAKASH VERMA *
}

\begin{abstract}
The effect of a conductive overburden on the electromagnetic response of a horizontal loop prospecting system to a tilted half-plane conductor is investigated by the analog modelling. It was found that the superficial conductive layer affects the anomaly by causing: a) appearance of an extra peak in the quadrature; b) base level shift of the profiles; c) phase rotation - consequently resulting in reversion of the quadrature -; and d) amplitude reduction. The appearence of an extra peak in the quadrature component complicates the quantification of the anomaly but, on the other hand, it may provide a useful information about the dip and induction number of the orebody as well as that of the overburden. The results assembled in the form of Argand diagrams can be used for a quantitative interpretation of an EM. anomaly to estimate the conductante, depth and dip of the conductor.
\end{abstract}

INTRODUÇÃo Os métodos eletromagnéticos (EM) têm sido largamente aplicados na prospecção de corpos de sulfetos e outros materiais condutivos. Um dos métodos EM mais utilizados é o Slingram, com as bobinas dispostas segundo o arranjo co-planar horizontal (HLEM). O campo magnético vertical secundário é captado no HLEM e dado em termos de porcentagem do campo primário gerado por um dipolo vertical (bobina horizontal).

A interpretação dos dados EM é comumente baseada nos resultados obtidos a partir de estudos de modelamento analógico (reduzido) ou numérico (digital). Tais estudos não consideram os efeitos devidos ao manto de intemperismo, à rocha encaixante e ao halo de sulfetos disseminados que se desenvolvem ao redor do corpo, por serem eles assumidos como altamente resistivos. 0 corpo condutivo, por sua vez, é simulado por modelos homogêneos e isotrópicos de geometria simples. Também é geralmente admitido que os minerais presentes não são magnéticos e inexistem relevos topográficos.

Os resultados obtidos com o estudo de situações extremamente simplificadas permitiram interpretações satisfatórias de vários levantamentos $\mathrm{EM}$, por exemplo, do Canadá e da Escandinávia (Ward 1971). Ao contrário, as interpreta* çбes de dados EM obtidos em várias outras áreas, como as brasileiras, conduziram a erros sérios (Parasnis 1971, Preston 1975, Palacky 1975, Lajoie \& West 1977, Palacky \& Kadekaru 1978, Palacky \& Sena 1979). As discrepâncias observadas são explicadas pelo não cumprimento das conđições estabelecidas no modelamento e merece especial atenção, tratando-se de regióes tropicais e semi-áridas, a não consideração de uma camada superficial levemente condutiva.

Os efeitos do manto de intemperismo sobre as anomalias EM mereceram alguns estudos, entre os quais se destaca o de Lowrie \& West (1965), realizado com a técnica HLEM e corpos condutivos tabulares (tipo semiplano) dispostos verticalmente. Os efeitos observados quando tal tipo de jazimento aparece inclinado são aqui apresentados.

\section{MODELAMENTO ANALÓGICO "EM"}

A resposta EM de uma situação real pode ser simulada em laboratório, em freqüência baixa, se uma quantidade adimensional conhecida como parâmetro de resposta $(\alpha)$ tiver o mesmo valor para os dois sistemas. No caso em estudo, $\alpha=\mu \omega \sigma t l$, em que $\mu=$ permeabilidade magnética; $\omega=$ frequêencia angular; $\sigma=$ condutividade elétrica; $t=$ espessura do corpo; e $l=$ distância entre as bobinas.

Modelos Corpos condutivos tabulares, como é o caso de filoes, podem ser aproximados a modelos do tipo semiplano por possuírem uma dimensão, a espessura, muito reduzida em relação às demais. $\mathrm{O}$ manto de intemperismo, por sua vez, guarda grande semelhança com modelos do tipo plano por possuir espessura também reduzida em comparação com sua distribuição areal.

$\mathrm{Na}$ simulação do corpo foram utilizadas folhas de aço inoxidável e de alumínio não magnéticas, de $1,00 \times 0,80 \mathrm{~m}$, e na do manto apenas folhas de aço também não magnéticas, de 2,00 x 1,00 m. Tais dimensões permitiram que as extremidades dos modelos pudessem ser mantidas a distâncias superiores a $0,75 l$ das bobinas, tornando desprezíveis os efeitos de borda dos mesmos. 0 corpo, com extensão superior a $2 l$, pode ser assumido como infinito em profundidade em relação à distância entre as bobinas (Lowrie \& West 1965).

$\mathrm{Na}$ tabela 1 são apresentadas as características físicas dos materiais utilizados, as freqüências de trabalho e os parâmetros de resposta obtidos para o corpo e para o manto (respectivamente, $\alpha_{\mathrm{c}} \mathrm{e} \alpha_{\mathrm{m}}$ ).

Os valores de condutividade foram determinados para tiras de $1,00 \times 0,01 \mathrm{~m}$ cortadas das folhas metálicas. Em cada tira foram feitas três medições com os eletrodos de potencial centralmente posicionados e separados de distâncias variáveis $(0,60 \mathrm{~m} ; 0,70 \mathrm{~m} \mathrm{e} 0,80 \mathrm{~m})$ e os eletrodos de corrente fixos às extremidades das tiras. As condutividades foram normalizadas para a temperatura ambiente do laboratório $\left(27,4^{\circ} \mathrm{C}\right)$.

Os valores de parâmetro de resposta refletem a restrita disponibilidade comercial de placas de diferentes espessuras bem como de materiais de custo acessível. Ainda assim, representam um grande número de situaçðes de campo, considerando-se as frequêencias $(100 \mathrm{~Hz}<\mathrm{f}<5.000 \mathrm{~Hz})$ e as distâncias entre as bobinas $(20 \mathrm{~m}<l<150 \mathrm{~m})$ comumente 
Tabela 1 - Caracteristicas/combinações das folhas metálicas usadas na simulação

\begin{tabular}{c|c|c|c|c}
\hline $\mathrm{f}(\mathrm{Hz})$ & Material & $\mathrm{t}\left(10^{-3} \mathrm{~m}\right)$ & $\sigma\left(10^{6} \mathrm{~S} / \mathrm{m}\right.$ & \multicolumn{1}{c}{$\alpha$} \\
\hline \multirow{6}{*}{325} & Aço & 0,283 & 1,377 & $0,25^{*}$ \\
\cline { 2 - 5 } & Aço & 2,570 & 1,418 & 2,21 \\
& Al & 0,290 & 33,367 & 6,21 \\
& Al & 0,428 & 32,126 & 8,82 \\
& Al & 0,709 & 33,097 & 15,05 \\
& Al & 1,513 & 33,658 & 32,67 \\
\hline \multirow{6}{*}{225} & Aço & 0,812 & 1,387 & $0,50^{*}$ \\
\cline { 2 - 5 } & Aço & 2,570 & 1,418 & 1,53 \\
& Al & 0,290 & 33,367 & 4,30 \\
& Al & 0,514 & 30,470 & 6,96 \\
& Al & 1,008 & 33,126 & 14,83 \\
& Al & 2,135 & 32,904 & 31,20 \\
\hline \multirow{4}{*}{450} & Aço & 0,812 & 1,387 & $1,00 *$ \\
\cline { 2 - 5 } & Aço & 2,570 & 1,418 & 3,06 \\
& Al & 0,290 & 33,367 & 8,60 \\
& Al & 0,514 & 30,470 & 13,91 \\
& Al & 1,008 & 33,126 & 29,66 \\
\hline
\end{tabular}

"Utilizado para simular o manto de intemperismo.

utilizadas, bem como valores reais de condutância do corpo e do manto de intemperismo. Neste sentido, a anátise de 72 valores de condutância obtidos em depósitos de sulfetos mostrou que $85 \%$ deles variam de 0,4 a $60 \mathrm{~S}$, sendo que, dentro desta faixa, $50 \%$ dos valores são inferiores a $10 \mathrm{~S}$ (Bosschart 1961, Paterson 1961, Strangway 1966, Questor Surveys Ltd. s.d.). A condutância do manto de intemperismo das regiốes brasileiras, com exceção da Amazônia, varia de 0,1 a is (Palacky \& Kadekaru 1978). Para aquela região, contudo, sabe-se que o manto pode mostrar espessuras consideráveis. Na Austrália, a condutância da camada superficial chega a atingir $10 \mathrm{~S}$, apesar de sua espessura centimétrica (Preston 1975).

Equipamentos $\mathrm{Na}$ figura $1 \hat{e}$ apresentado o diagrama de blocos dos sistemas de energização e de recepção utilizados.

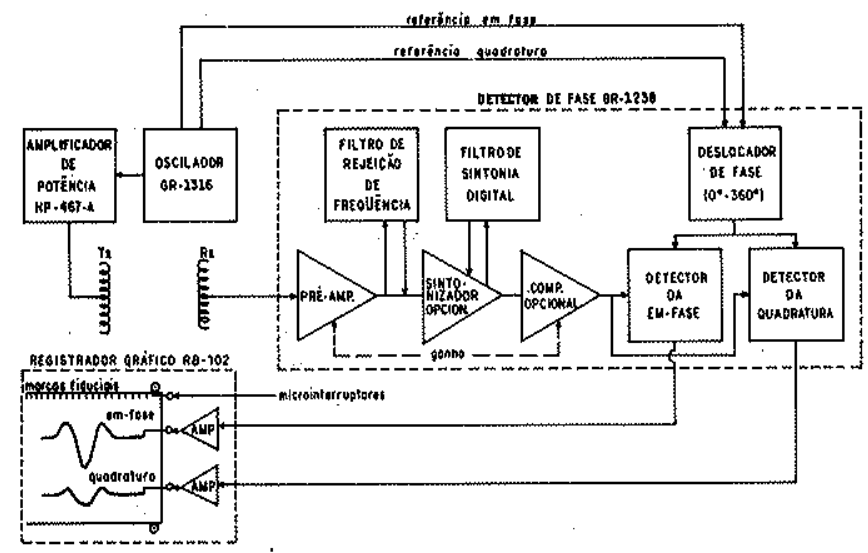

Figura 1 - Diagrama de blocos dos sistemas de energização e recepção
Um oscilador de frequêencia de $10 \mathrm{~Hz}$ a $100 \mathrm{kHz}$ (General Radio, USA) fornece um sinal a uma bobina com $0,025 \mathrm{~m}$ de diâmetro e núcleo de ferrite, através de um amplificador de potência de 10 W (Hewlett-Packard, USA). A bobina transmissora, apesar de sua dimensão relativamente exagerada considerando-se o fator de escala do modelamento, representa um dipolo magnético porque seu diâmetro é muito menor que a separação de $0,25 \mathrm{~m}$ utilizada entre as bobinas.

$O$ sinal transmitido é detectado indutivamente por uma bobina receptora de dimensão igual à da bobina transmissora com núcleo também de ferrite. Segue então para um detector de fase (General Radio, USA), onde é analisado em termos das componentes em fase e em quadratura em relação a um sinal de referência enviado pelo oscilador. Esse sinal é, antes das medições, calibrado de modo a ser igual em fase e amplitude à f.e.m. (força eletromotriz) gerada pelo campo primário.

Os resultados, dados como porcentagem do campo primário, seguem para um registrador gráfico de dois canais (ECB, BR). 0 registrador conta também com um marcador de eventos que permite o posicionamento das medidas obtidas em relação à disposição do sistema de bobinas sobre o modelo.

Um sistema mecânico construfdo em madeira e acrílico permite posicionar o corpo a diferentes profundidades com variados mergulhos e o manto, horizontalmente. Ao longo de trilhos desloca-se, movido por um motor passo-a-passo, um carrinho de acrílico contendo as bobinas. Dezenove microinterruptores dispostos a cada $0,05 \mathrm{~m}$ do trilho são acionados pelo carrinho em movimento e fornecem marcas de posicionamento ao registrador.

Medições As medições foram realizadas de forma contínua ao longo de perfis perpendiculares à direção do corpo, calibrando-se o sinal de referência sobre o manto e fora do mesmo. Esta última calibração, utilizada no trabalho de Lowrie \& West (1965), é na prática mais dificilmente executada. Havendo o manto de intemperismo na região sob investigação, a calibração será feita sobre o mesmo. Esta raramente poderá ser realizada numa posição em que o manto não ocorra, ou seja, onde afloram rochas resistivas.

No caso $\alpha_{\mathrm{m}}=0,25$, foram realizadas mediçoes unicamente com a calibração na ausência do manto. Testes executados mostraram que os resultados obtidos com as duas diferentes calibraçбes são extremamente próximos e, por outro lado, que a calibração sobre o manto é mais sensível a variaçóes de altura do modelo usado na simulação do manto (Silva 1981). Essa altura foi mantida constante e igual, quando normalizada com respeito à distância entre as bobinas $(\mathrm{hm} / \mathrm{l})$, a 0,10 . Para o corpo foram consideradas as seguintes profundidades normalizadas $\left(\mathrm{h}_{\mathrm{c}} / l\right): 0,20,0,30$ e 0,50 . Ainda, mergulhos $(\theta)$ de $90^{\circ}, 75^{\circ}, 60^{\circ}, 45^{\circ}$ e $30^{\circ}$. Para efeito de comparação, todas as medições realizadas foram repetidas fazendo-se $\alpha_{\mathrm{m}}=0$, isto é, assumindo-se a inexistência da cobertura superficial condutiva.

EFEITOS DO MANTO DE INTEMPERISMO OS resultados obtidos mostram que as anomalias geradas por corpos condutivos do tipo semiplano podem sofrer grandes modificações quando está presente o manto de intemperismo (Fig. 2), tais como: a) aparecimento de um pico extra na quadratura; b) deslocamento do perfil em relação à base; c) rotação da fase (podendo gerar a reversão da quadratura); e d) redução da amplitude da anomalia. 


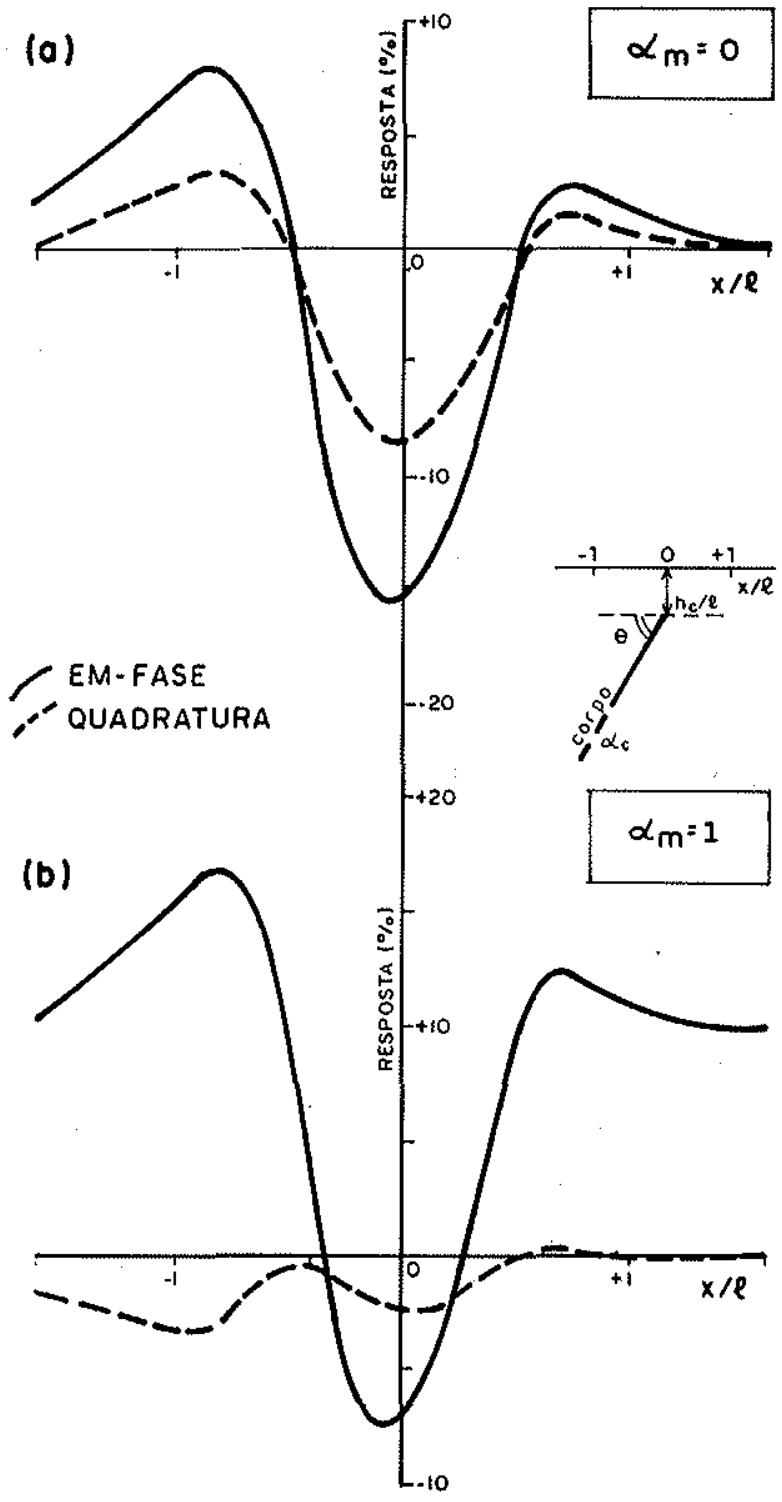

Figura 2 - Perfis obtidos sobre um condutor tabular inclinado $\left(\alpha_{\mathrm{c}}=15, \theta=60^{\circ} \mathrm{e} h_{c} / 1=0,3\right):$ a. quando inexiste $o$ manto de intemperismo $\left(\alpha_{m}=0\right)$; $e$ b. na presença do mes$m o\left(\alpha_{m}=1\right.$, calibração realizada fora da cobertura)

Todos esses efeitos, com exceção do aparecimento de um pico extra na quadratura, também foram observados para corpos verticais (Lowrie \& West 1965).

Aparecimento de um pico extra na quadratura $O$ aparecimento do pico extra na componente em quadratura observada sobre um corpo capeado pelo manto de intemperismo está intimamente relacionado à diminuição do ângulo de mergulho do corpo (Fig. 3a). Entretanto, para as situações estudadas, observou-se que tal efeito é favorecido pelo aumento tanto do parâmetro de resposta do manto quanto daquele do corpo (Figs. $3 b$ e $3 c$, respectivamente). Este efeito é conseqüência da maior interação entre o corpo $\mathrm{e}$ o manto, devido à maior proximidade dos mesmos promovida pela diminuição do mergulho do corpo. Corrobora nesse sentido a redução ou mesmo o desaparecimento deste efeito com o aumento da profundidade do corpo (Fig. 3d).
Na componente em fase não foi observado este tipo de efeito para os valores de $\alpha_{c}$ e $\theta$ considerados na investigação.
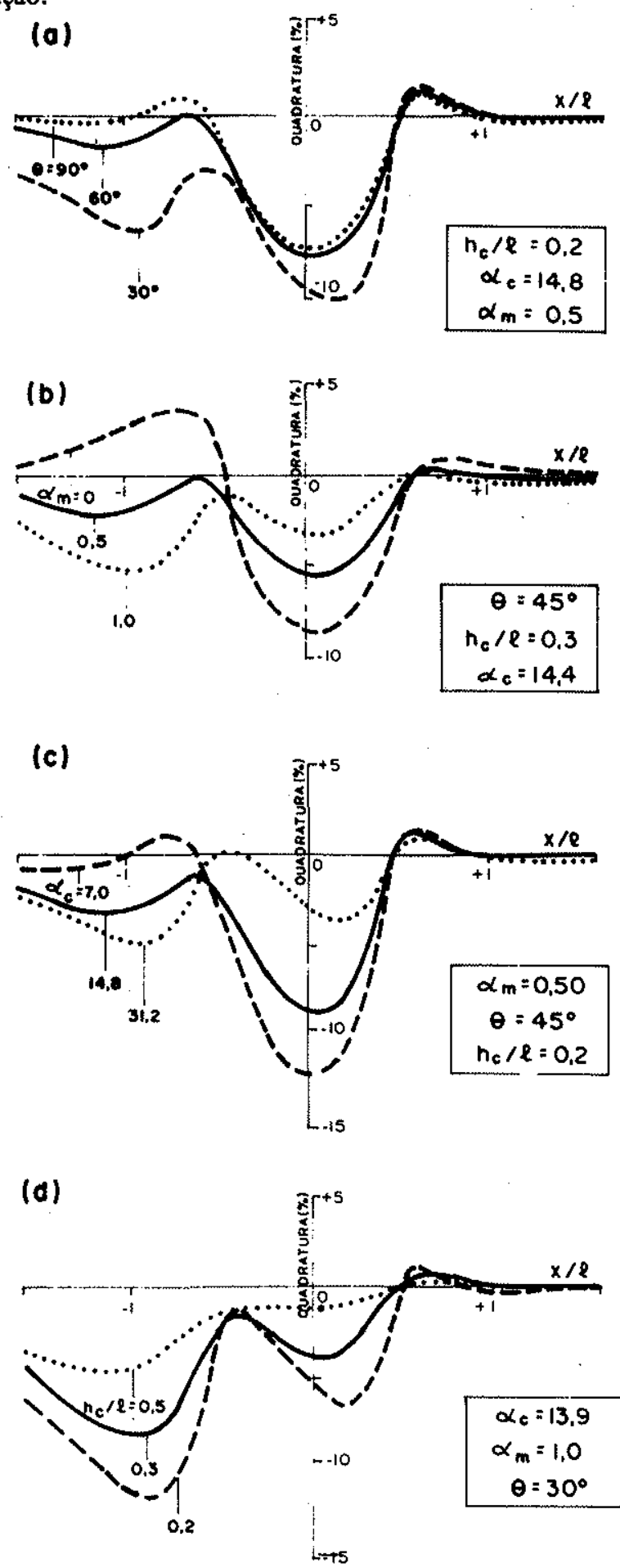

Figura 3 -.Aparecimento de um pico extra na quadratura com a variação dos parâmetros: a. mergulho do corpo ( $\theta)$; b. parâmetro de resposta do manto $\left(\alpha_{m}\right)$; c. parâmetro de resposta do corpo $\left(\alpha_{c}\right) ;$ e d. profundidade do corpo $\left(h_{c} / 1\right)$

Deslocamento do perfil em relação à base $O$ desloca. mento do perfil em relação à base $(0 \%)$, promovido pela presença do manto, ocorre unicamente quando a calibração é feita na ausência deste último. 
$O$ deslocamento do perfil em fase cresce com o aumento do parâmetro de resposta do manto, para a faixa dos valo. res investigados (Fig. 4a). 0 mesmo ocorre com o perfil da quadratura para pequenos valores de $\alpha_{\mathrm{m}}$ mas, com o incremento deste último, o deslocamento do perfil atinge valo. res negativos (Fig. 4b).

$O$ efeito em questã́o, consequêencia da rotação de fase provocada pelo manto considerado de forma isolada, depende do parâmetro de resposta deste modelo bem como da altura em que é posicionada a folha metálica utilizada em sua simulação (Fig. 5).

Rotação da fase e redução da amplitude da anomalia $\quad$ manto de intemperismo promove a rotação da fase da anomalia no sentido horário. Como conseqüência, a componente em quadratura pode aparecer reversa. Os casos estudados indicam que a reversão da quadratura é favorecida pelo
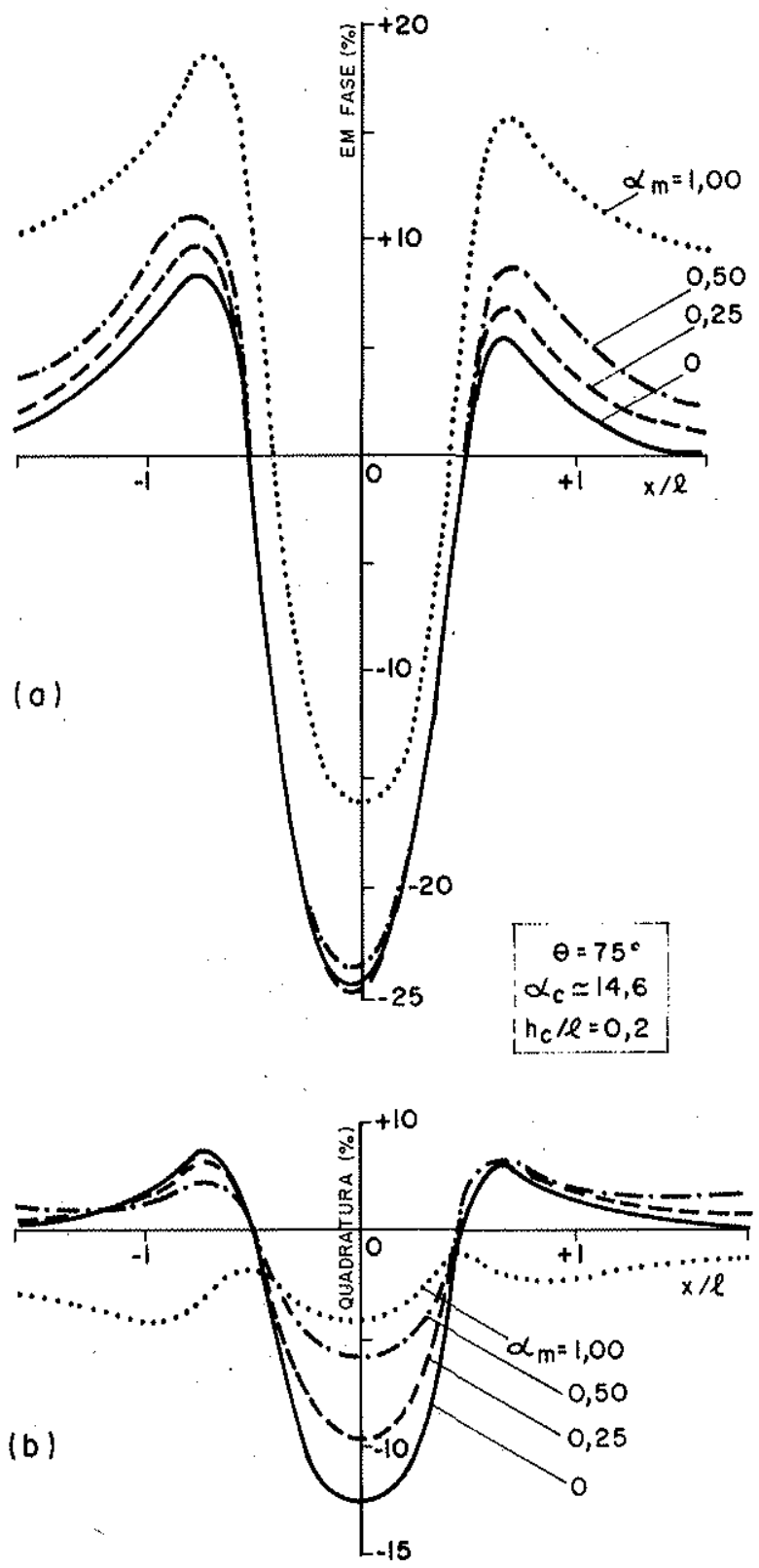

Figura 4 - Deslocamento do perfil em relação à base (0\%) das componentes; a. em fase e b. em quadratura aumento do parâmetro de resposta tanto do manto quanto do corpo (Figs. 6a e 6b, respectivamente).

$\mathrm{O}$ efeito de rotação da fase da anomalia bem como o de redução de sua amplitude são mais bem percebidos pelos diagramas de Argand. Na construção de tais diagramas foram utilizados os valores pico-a-pico das componentes, tomados nos lados a favor e contrário ao mergulho do corpo (Fig. 7a). O procedimento adotado, além de evitar imprecisões devidas a desvios eventuais da base $0 \%$, permite a quantificação das anomalias obtidas com a calibração fora do manto e, por isso mesmo, deslocadas com respeito a $0 \%$.

A quantificação da anomalia pode ser uma tarefa problemática quando se desenvolve o pico extra (Figs. $7 \mathrm{~b}$ e $7 \mathrm{c}$ ). Em tais casos, a quadratura foi considerada reversa quando seu pico "positivo" do lado a favor do mergulho do corpo

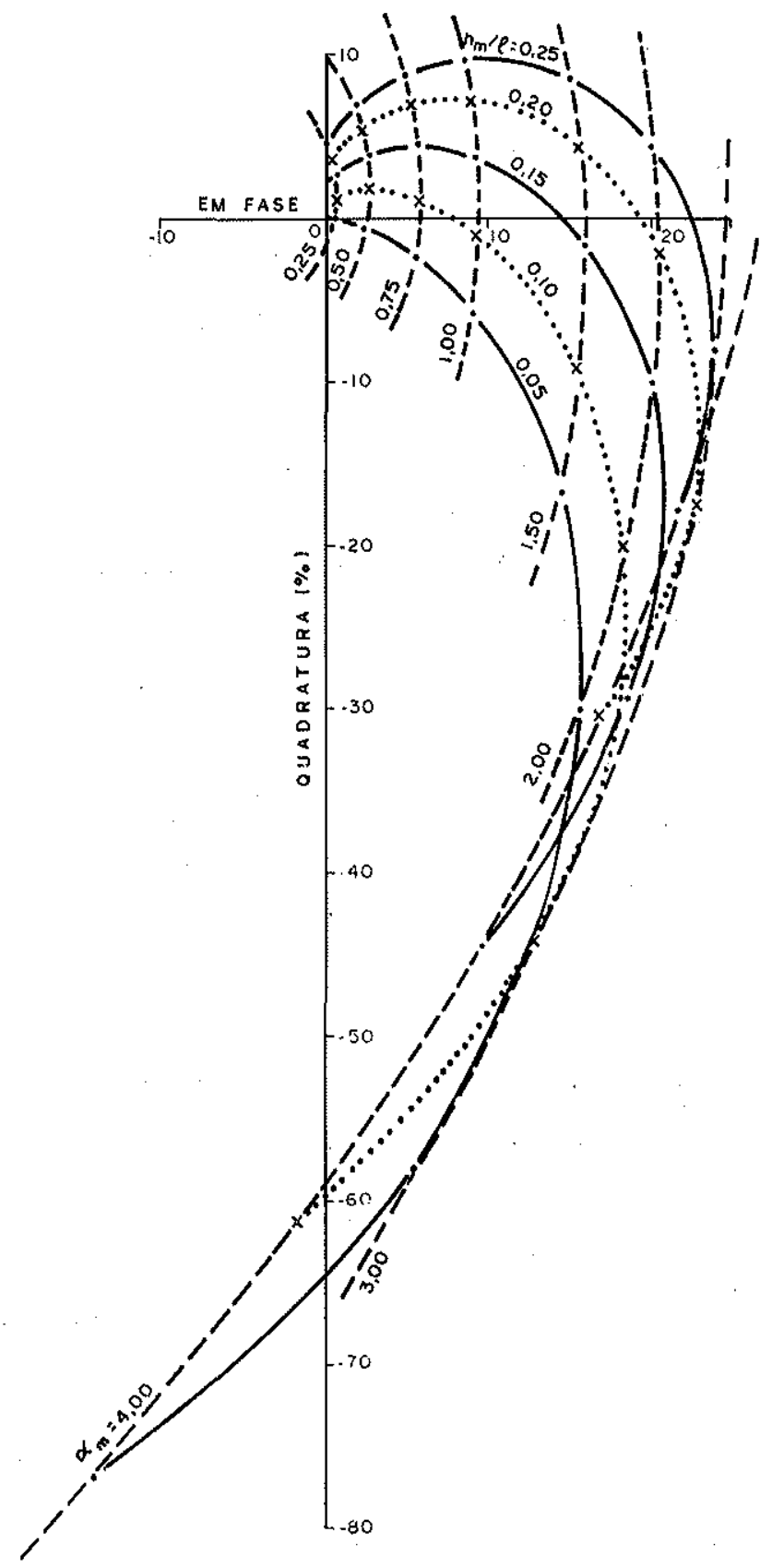

Figura 5 - Respostas do manto de intemperismo 

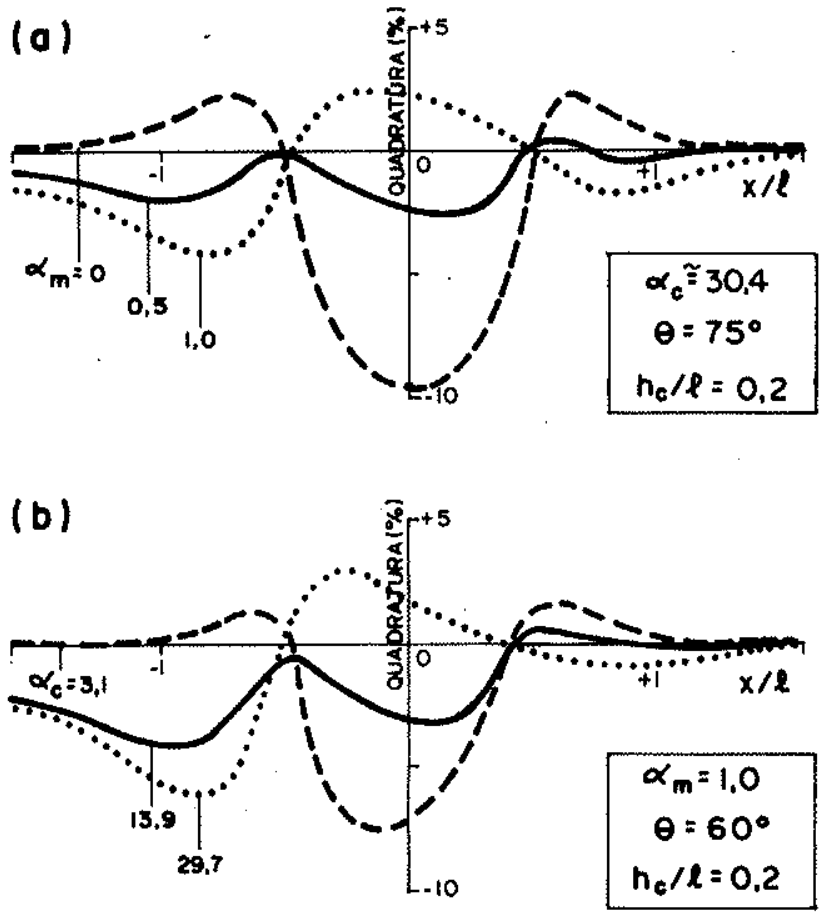

Figura 6 - Reversão da quadratura com o aumento dos parâmetros de resposta: a, do manto $\left(\alpha_{m}\right)$ e b. do corpo $\left(\alpha_{c}\right)$

(lado este que no perfil é indicado pela componente em fase) atinge valor absoluto superior ao do pico "positivo" que se desenvolve no outro lado (apesar do termo "positivo", ambos os picos considerados podem atingir valores abaixo da base $0 \%$ ).

$O$ procedimento de quantificação adotado apóia-se na observação de que, com o aumento da profundidade do corpo, o pico extra diminui e a reversão, existindo, torna-se mais nítida (Figs. $7 b_{1}$ e $7 c_{1}$ ). Assim, assumir que o perfil mostrado na figura $7 \mathrm{~b}$ não tem quadratura reversa concorda com o que é indicado por seu correspondente profundo (Fig. $7 b_{1}$ ). Da mesma forma ocorre com o perfil ilustrado na figura $7 \mathrm{c}$, considerado reverso $\mathrm{e}$, portanto, em consonância com os resultados verificados para uma maior profundidade do corpo (Fig. $7 c_{1}$ ).

Por outro lado, os valores pico-a-pico determinados pelo procedimento adotado, quando utilizados na construção de diagramas de Argand, produzem para as situaçóes em que $\alpha_{m}$ e relativamente alto curvas descontínuas para uma mesma profundidade (Fig. 8). Contudo outros procedimentos de quantificação mostraram maiores desvantagens, como, por exemplo, curvas com revoluçōes sobre si mesmas sem guardarem entre si uma mesma tendência. Esta é uma situação muito mais complexa que a observada na figura 5 e, portanto, de pouca valia para uso na interpretação de dados reais.

Os diagramas construídos mostram que o ângulo de rotação da fase e a porcentagem de redução da amplitude da anomalia:

- são diretamente proporcionados à $\alpha_{\mathrm{m}}$ (Figs. 8 e 9);

- são maiores para os valores pico-a-pico a favor (Figs. 9 e 10); e

- à medida que o corpo se afasta da vertical, diminuem se forem considerados os valores pico-a-pico contra (Figs. 9 e
11) e aumentam para os pico-a-pico a favor (Figs. 10 e 12).

Para corpo vertical e $\alpha_{\mathrm{m}}=0,25 ; 0,50$ e 1,00; o ângulo de rotação da fase gira em torno de, respectivamente, $6,25^{\circ}$; $12,5^{\circ}$ e $25^{\circ}$, concordando com os resultados de Lowrie \& West (1965). Para corpos inclinados, esse ângulo varia em torno de $\pm 2,0^{\circ}$ dos valores encontrados para o corpo vertical.

As reduçôes da amplitude são, para $\alpha_{\mathrm{m}}=0,25 ; 0,50 \mathrm{e}$ 1,00 ; iguais ou pouco inferiores a, respectivamente, $2,5 \%$; $5,0 \%$ e $10,0 \%$; considerando-se os perfis obtidos com a calibração na ausência do manto. Para os demais, as reduções de amplitude podem ser bastante superiores às apresentadas (Fig. 13).

Exceções às tendências descritas refletem as distorções encontradas em alguns dos diagramas, produzidas pelos perfis de pico extra bastante desenvolvido e em franca oposição à reversão da componente em quadratura, ou seja, obtidos para valores elevados de $\alpha_{c}$ e, principalmente, $\alpha_{\mathrm{m}}$ bem como pequenos $\theta$. Um bom exemplo a esse respeito é dado pela redução do ângulo de rotação da fase para valores elevados de $\alpha_{\mathrm{c}}$, antes de se dar a reversão da quadratura. Assim, o pico extra é percebido nos diagramas apenas de forma indireta. O mesmo não ocorre com a reversão da quadratura, que pode ser observada diretamente nos diagramas, assim como seu favorecimento pelo aumento dos parâmetros de resposta do manto e do corpo, notando-se que ela ocorre qualquer que seja o mergulho do corpo (Figs. 8 a 13).

CONCLUSÓES O campo magnético sofre, em cada metro que atravessa do manto, uma rotação e uma atenuação de, respectivamente, $180^{\circ} / \delta$ e $(1 / \mathrm{e}) \times \delta$ de seu valor original, sendo $\delta=$ skin depth. 0 mesmo ocorre com o campo que alcança a bobina receptora após atravessar o manto. As respostas obtidas com a calibração sobre o manto, que compensa as rotaçōes e atenuações por ele geradas, mostram praticamente a mesma rotação daquelas levantadas com o outro tipo de calibração, que não promove as referidas compensações, bem como uma atenuação supeior a destas últimas (Fig. 13). A resposta HLEM de corpos do tipo semiplano capeados por manto de intemperismo constitui, portanto, um problema bastante difícil por envolver não só o efeito adicional do manto como também complexa interação entre este último e o corpo.

$O$ aparecimento do pico extra na componente em quadratura, estritamente ligado com a diminuição do mergulho do corpo e, portanto, maior proximidade entre este e o manto, corrobora a existência de importante interação entre os dois condutores. Até $\alpha_{\mathrm{m}}=1$, não foram observados efeitos análogos na componente em fase.

$O$ pico extra da quadratura complica bastante a quantificação das anomalias, notadamente quando estas se encontram nas vizinhanças da reversão. Por outro lado, para as situações investigadas, esses dois efeitos funcionam como indicadores qualitativos dos parâmetros dos condutores. Assim, têm-se as seguintes simples regras para a interpretação qualitativa das anomalias geradas por corpos do tipo semiplano:

I) A existência de pico extra na quadratura denota a presença de corpo condutivo de mergulho suave e parâmetro de resposta significativo, bem como a existência de manto de intemperismo de influência não despre. zível. Quanto mais evidente for o pico extra, mais raso pode estar o corpo. 
(a)
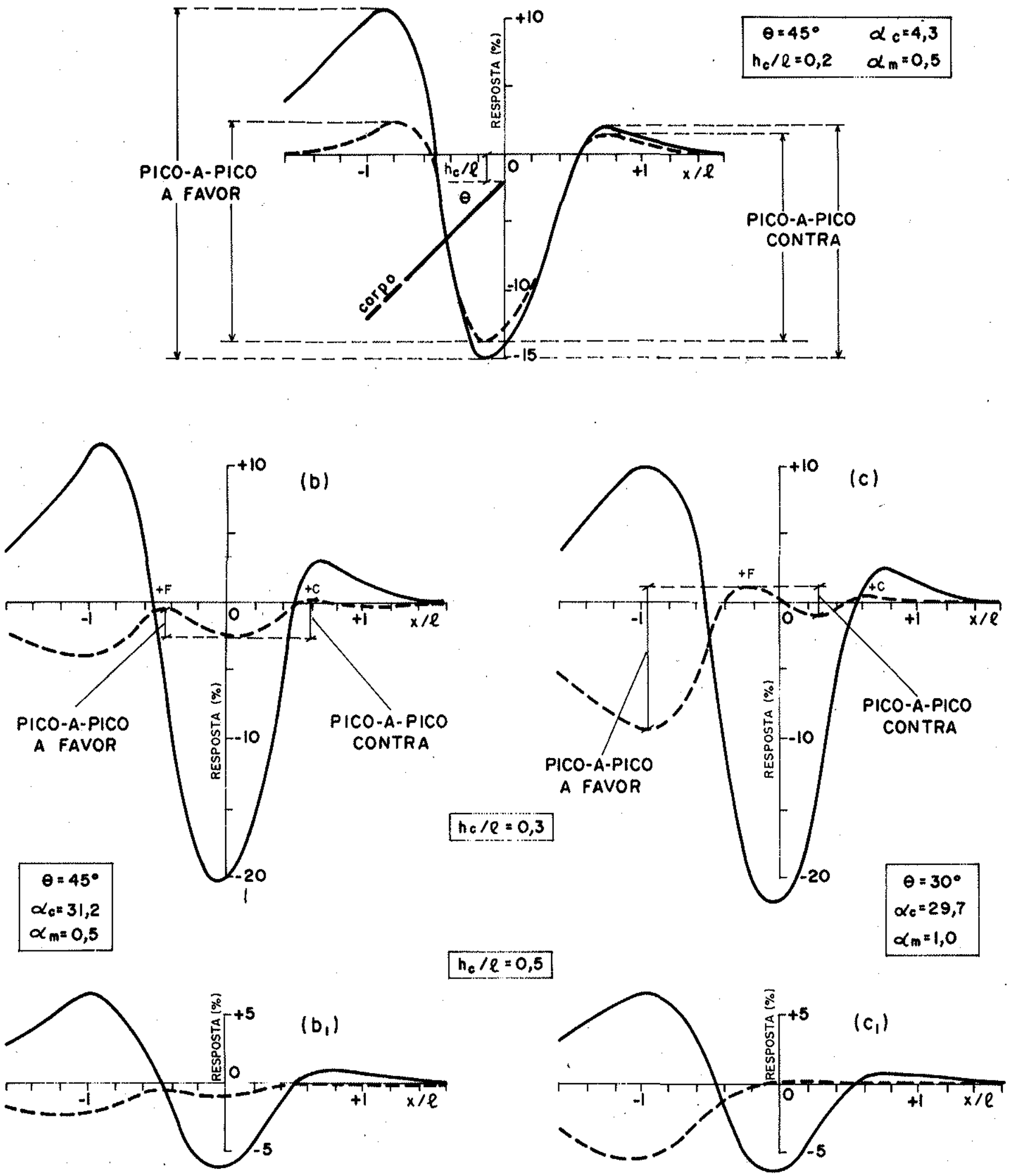

$$
\begin{aligned}
& \text { EM FASE QUADRATURA-- } \\
& \text { PICO "POSITIVO" DO LADO: A FAVOR +F } \\
& \text { CONIRA +C }
\end{aligned}
$$

Figura 7 - Quantificaçäo das anomalias: a. perfil simples; $b_{\text {. }}$ perfil complexo sem quadratura reversa; $\mathrm{b}_{\mathrm{i}}$. correspondente profundo do perfil (b); c. perfil complexo com quadratura reversa; $e \mathrm{c}_{1}$, correspondente profundo do perfil $\mathrm{c}$ 


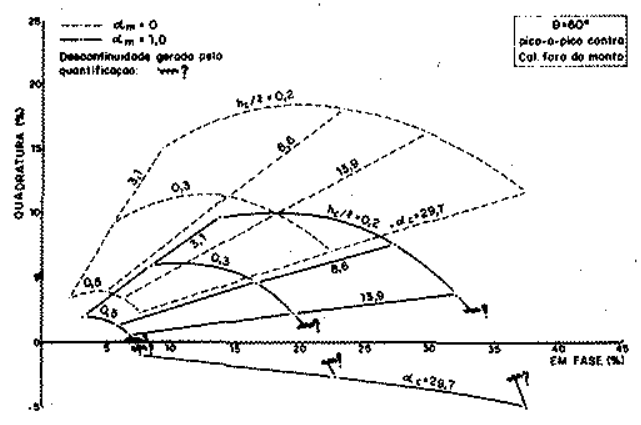

Figura 8 - Diagramas de valores pico-a-pico do lado contrário ao mergulho do corpo $\left(60^{\circ}\right)$ para $\alpha_{m}=0$ e 1,00 (calibraçäo fora do manto)

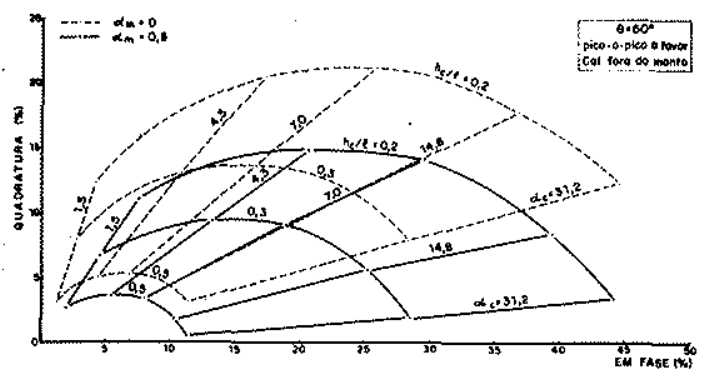

Figura 10 - Diagramas de valores pico-a-pico do lado a favor do mergulho do corpo $\left(60^{\circ}\right)$ para $\alpha_{m}=0$ e 0,50 (calibraçäo fora do manto)

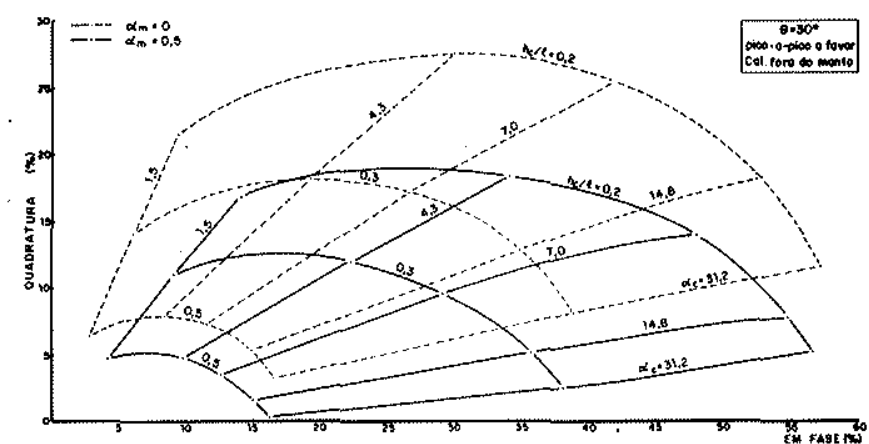

Figura 12 - Diagramas de valores pico-a-pico do lado a favor do mergulho do corpo $\left(\theta=30^{\circ}\right)$ para $\alpha_{m}=0$ e 0,50 (calibração fora do manto)

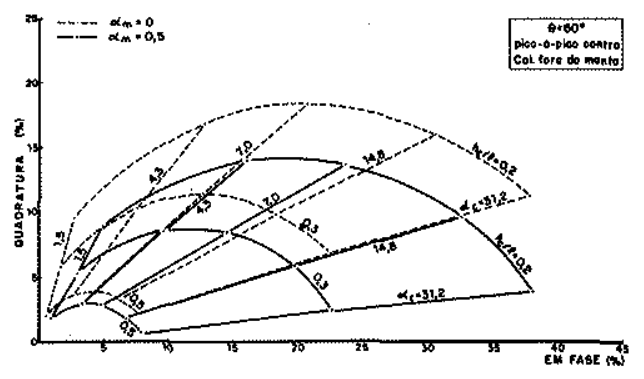

Figura 9 -Diagramas de valores pico-a-pico do lado contrário ao mergulho do corpo $\left(60^{\circ}\right)$ para $\alpha_{m}=0$ e 0,50 (calibraçäo fora do manto)

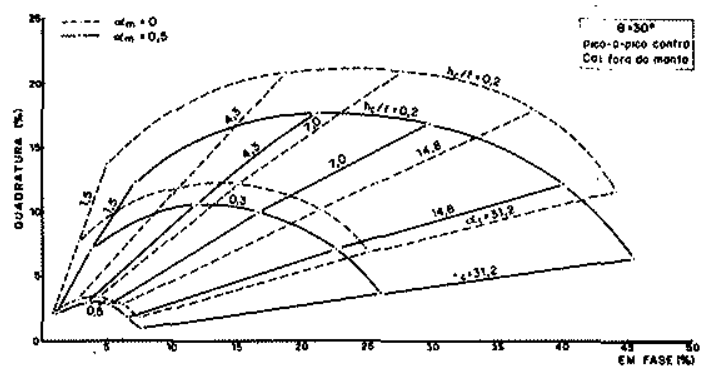

Figura 11 - Diagramas de valores pico-a-pico do lado contrário ao mergulho do corpo $\left(30^{\circ}\right.$ ) para $\alpha_{m}=0$ e 0,50 (calibração fora do manto)

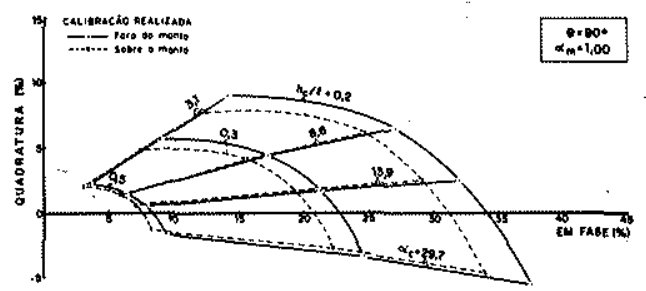

Figura 13 - Diagramas de valores pico-a-pico para corpo vertical, $\alpha_{m}=1$ (ambas as calibraçōes) 
II) A reversão da quadratura indica a presença de corpo condutivo capeado por manto de intemperismo, ambos com significativo parâmetro de resposta.

Os trechos dos perfis com anomalia, que contenham pelo menos um desses efeitos, não devem ser abandonados, não obstante $o$ aspecto desanimador que a referida anomalia pode alcançar, por exigir quantificação não tão simples, bem como por exibir feiçбes que podem ser confundidas com sintomas de perturbações/ruídos. O detalhamento desses trechos, com diferentes frequêencias e espaçamentos entre as bobinas, permitirá uma melhor compreensão do significado da anomalia inicialmente detectada. Esse procecimento também facilitará a interpretação quantitativa, pois permite a obtenção de respostas com larga dominação de um dos efeitos em questão, de preferência a reversão, que não difículta a execução de tal tarefa.

A aplicabilidade dos resultados pode ser ilustrada com as respostas HLEM obtidas sobre um corpo de pirita vertical, capeado por uma cobertura superficial de condutância $(\sigma t)$ igual a $0,75 \mathrm{~S}$, localizado em Abititi Clay Belt, Canadá (Lajoie \& West 1977).
Os perfis levantados mostram, com o aumento da freqüência, um deslocamento com respeito à base $(0 \%)$ e a reversão da componente em quadratura, efeitos indicativos da presença da cobertura condutiva. $O$ deslocamento dos perfis, em adicional, mostra que a calibração das bobinas foi realizada onde os efeitos do manto são inexpressivos (Fig. 14a). Os resultados da quantificação do pico negativo das anomalias (comportamento semelhante aos valores pico-a-pico) - lançados em diagrama de Argand construído por Nair et al. (1968) para corpo vertical e $\alpha_{\mathrm{m}}=0-$ migram de forma substancialmente diferente da esperada teoricamente (Fig. 14b). Tal comportamento é, contudo, idêntico ao observado nos diagramas aqui apresentados, construídos para $\alpha_{\mathrm{m}} \neq 0$ (Figs. 8 a 13 ).

Agradecimentos $\mathrm{O}$ presente trabalho foi realizado graças ao suporte financeiro concedido pela FINEP e CNPq, e à bolsa de estudo fornecida ao autor principal pela Pronuclear. A estas entidades, os autores agradecem o apoio proporcionado.

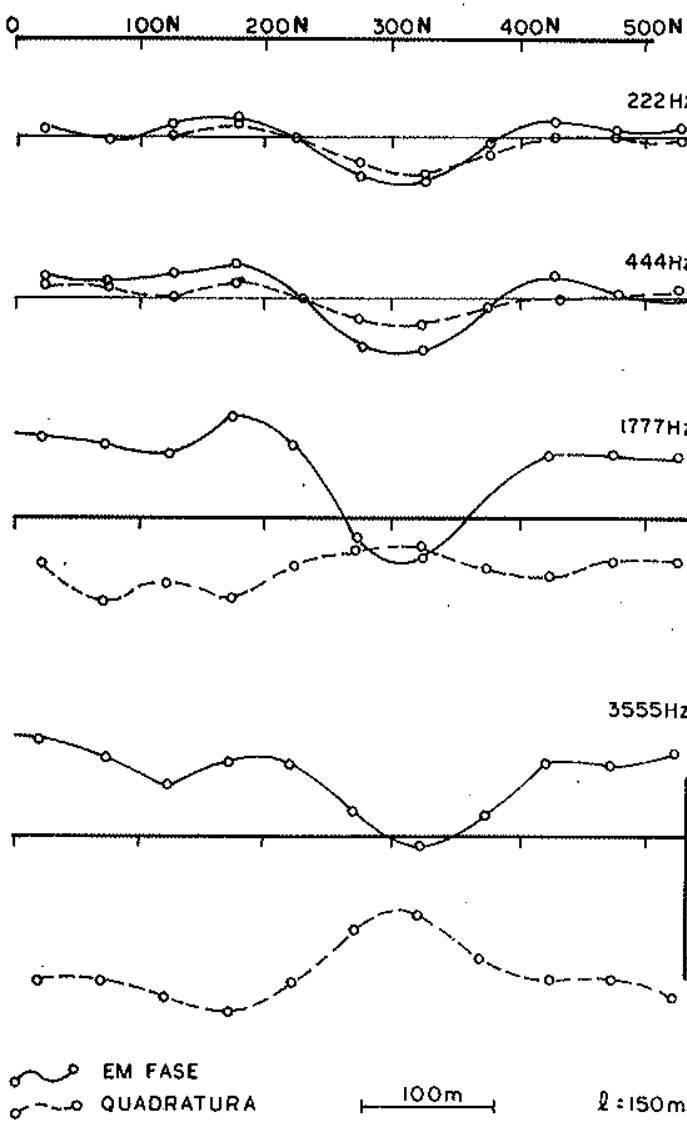

(a)
OT Q QUAORATURA $\quad 100 \mathrm{~m} \quad l=150 \mathrm{~m}$

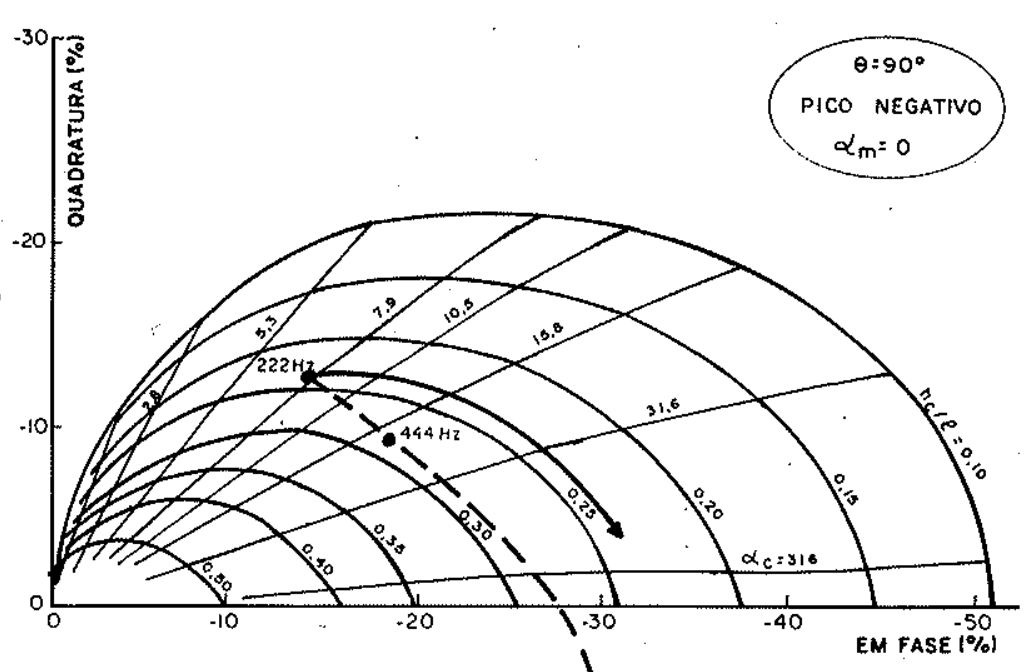

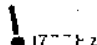

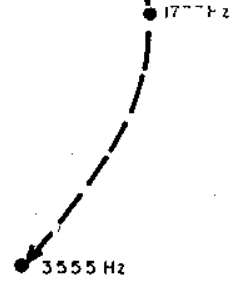

$\underset{\text { TRAJETÓRIA TEÓRICA }}{\longrightarrow}$

(b)

Figura 14 - a Perfis de campo sobre corpo de sulfetos (Canadá) e b. migração (observada e teórica) dos valores do pico negativo (após Lajoie \& West 1977)

\section{REFERENCIAS BIBLIOGRAFICAS}

BOSSCHART, R.A. - 1961 - On the occurrence of low resistivity geological conductors. Geoph. Prosp., 9(2):203-212.

LAJOIE, J.J. \& WEST, G.F. - 1977 - Short note: two selected field examples of EM anomalies in a conductive environment. Geoph, 42(3):655-660.
LOWRIE, W. \& WEST, G.F. - 1965 - The effect of a conducting overburden on electromagnetic prospecting measurements. Geoph., 30(4):624-632.

NAIR, M.R.; BISWAS, S.K.; MAZUMDAR, K. - 1968 - Experimental studies on the electromagnetic response of tilted 
conducting half-planes to a horizontal-loop prospecting system. Geoexpl., 6(4):207-244.

PALACKY, G.J. - 1975 - Interpretation of Input AEM measurements in areas of conductive overburden. Geoph., 40(3): 490-502.

PALACKY, G.J. \& KADEKARU, K.-- 1978 - Efeito do intemperismo tropical nos levantamentos eletromagnéticos. In: CONGR. BRAS. GEOL., 30, Recife, 1978. Anais..., Recife, SBG. v. 5, p. 2323-2330.

PALACKY, G.J. \& SENA, F.O. - 1979 - Conductor identifica* tion in tropical terrains - Case histories from the Itapecuru Greenstone belt, Bahia, Brazil, Geoph., 44(12):1941-1962.

PARASNIS, D.S. - 1971 - Analysis of some multi-frequency, multi-separation electromagnetic surveys. Geoph. Prosp., 19(2):163-179.

PATERSON, N.R. - 1961 - Experimental and field data for the dual frequency phase-shift method of airborne electromagnetic prospecting. Geoph., 26(5):601-617.

PRESTON, B. - 1975 - Review - Difficulties for the electromagnetic method in Australia. Geoexpl, 13(1):29-43.
QUESTOR SURVEYS Ltd. - s.d. - The Questor Method. Ontario, Questor Surveys Ltd. s.p. (case histories: Brouillan Discovery, Detour River Discovery, Lessard Deposit, Magusi River Discovery, New Insco Discovery, Rail Zone, Reed Lake Discovery, Thierry Deposit, Yava Zone).

SILVA, L.M.C. - 1981 - Efeitos do manto de intemperismo sobre anomalias eletromagnéticas provocadas por corpos tabulares inclinados - Um estudo através de modelamento reduzido. Belém (Dissertação de Mestrado, Centro de Geociências UFPa), $115 \mathrm{p}$.

STRANGWAY, D.W. - 1966 - Electromagnetic parameters of some sulfide ore bodies. In: HANSEN, D.A. et al, eds. Mining geophysics. USA, SEG. v. 1, p. 227-242.

WARD, S.H. - 1971 - Foreword and introduction. Geoph., 36(1): $1-8$.

MANUSCRITO

Recebido em 01 de abril de 1986

Revisão aceita em 23 de outubro de 1986

No teste de hipóteses reside a diferença primordial entre o pesquisador e o teórico. 0 primeiro procura diligentemente pelos fatos que podem invalidar sua teoria tentativa, o outro fecha seus olhos para estes e pesquisa apenas por aqueles (fatos) que a irão sustentar.

G. Gilbert, 1885, Am. J. Sci., 31 (181-186) p. 286 